\title{
Between surveillance and subjectification: the developing governance of quality and safety in English hospitals
}

\author{
Graham P. Martin, Myles Leslie, Joel Minion, Janet Willars \& Mary Dixon-Woods
}

\begin{abstract}
Two understandings of the dynamics of power developed by Foucault have been extensively used in analyses of contemporary healthcare: disciplinary power and governmentality. They are sometimes considered alternative or even contradictory conceptual frameworks. Here, we seek to deploy them as complementary ways of making sense of the complexities of healthcare organisation today. We focus on efforts to improve quality and safety in three UK hospitals. We find a prominent role for disciplinary power, including a panoptic gaze that is to some extent internalised by professionals. We suggest, however, that the role of disciplinary power relies for its impact on complementary strategies that are more akin to governmentality. These strategies foster organisational contexts that are receptive to disciplinary work. More fundamentally, we find that both disciplinary power and governmentality work on subjectivities in rather a different manner from that suggested by conventional accounts. We offer an alternative, less individualised and more socialised, understanding of the way in which power acts upon subjectivity and behaviour in professional contexts.
\end{abstract}

Published in: $\quad$ Social Science \& Medicine 99: 80-88 http://www.sciencedirect.com/science/article/pii/S0277953613005662 doi:10.1016/j.socscimed.2013.10.018

\section{Introduction}

Contemporary healthcare systems in the industrialised world are characterised by a curious mix of governance regimes. On the one hand, recent technological and managerial developments have created renewed possibilities for so-called managerialism-in the form of surveillance of professional practice and micro-level control-that were previously unimaginable. New information-technology (IT) systems and other techniques of surveillance seek to open clinical practice to the gaze of managers in a way that has been characterised by some authors (e.g. Timmons, 2003; Waring, 2007) in terms of Michel Foucault's (1979) notion of disciplinary power, and its work to make visible and regulate individuals' conduct.

Concurrently, however, these very managerialist logics are frequently characterised as having been at least partially displaced by a host of alternative approaches to guiding the behaviour of practitioners, professions and organisations. Developments such as network governance (Rhodes, 2007), the reinvigoration of professionalism as a mode of social control (Martin et al., 2012) and 'post-bureaucratic' forms of influence such as 'leadership' and 'engagement' (Martin \& Learmonth, 2012) highlight a renewed recognition of the limitations of top-down hierarchy, and the rise of alternative forms. This analysis of the exercise of influence in 
healthcare organisations resembles a form of power that appeared slightly later in Foucault's (e.g. 1991) work: 'governmentality'. Building on sketches in Foucault's last interviews, lectures and books, scholars of neoliberal governmentality describe a world where power is exercised through distributed technologies and discourses that act on individuals' own freedom: their decisions, their creativity, their relationships with others. Governmentality has been used widely to analyse how power acts upon subjectivities in various contexts, including healthcare (e.g. Ferlie, McGivern, \& Fitzgerald, 2012; Sheaff et al., 2004).

Discipline and governmentality are not totally distinct regimes of power: some authors argue that the latter is a continuation of the former (e.g. Elden, 2002). Nevertheless, other authors highlight tensions between the two, noting that they act on their subjects in potentially conflicting ways. Yet to date few studies have attempted to deploy these two conceptual frameworks of Foucault in tandem, or explore their contradictions and complementarities. Further, the frameworks have often been used as macro-level heuristics for understanding overarching power regimes; largely lacking from studies in healthcare has been a critical engagement with Foucault as a means of understanding the micro-level operation of technologies of power.

This paper seeks to address these two deficits by drawing on discipline and governmentality to understand the operation of the governance of healthcare quality and safetya field which typifies the 'mixed economy' of technologies of power of contemporary organisation. We draw on interviews with senior and middle managers in UK hospitals about the strategies they use to address quality and safety and the impact of these on clinical-professional behaviour. We note overlaps, tensions and synergies between regimes of power, but suggest above all that they are interdependent-and that they rely on fostering forms of agency anticipated in Foucault's writings, but under-acknowledged and under-analysed by most Foucaultian scholars since. We begin by examining in more detail Foucault's theories of power, the overlaps and tensions between them, and how they have been deployed in analyses of the contemporary governance of healthcare professionals' behaviour.

\section{Foucault, regimes of power, and healthcare governance}

Much of Michel Foucault's work recounts a history of how human subjectivities are constituted through shifting regimes of power and changing agencies, discourses and relationships. From the 1970s, Foucault became increasingly concerned with what he termed 'disciplinary power': the process whereby individuals are divided and ordered by external forces (e.g. Foucault, 1979), and whereby such forces are internalised by those individuals. Slightly later, Foucault put forward the notion of 'governmentality'. This sees subjects as constituted less by the internalisation of an external disciplining gaze, and more through coming to recognise and animate their own selves, and their identities, purposes and desires-but under the influence of powerful discourses that guide them in these decisions (e.g. Foucault, 1982). We consider each concept in turn.

\section{Second-level heading: Garamond 12 pt italic, 6 pt gap before, 3 pt gap after}

Foucault (e.g. 1979, 1988) traced the rise of 'disciplinary power', found initially in the enclosed institutions (schools, prisons, hospitals) of the seventeenth-to-eighteenth centuries. This was power quite different from the power of a sovereign ruler over juridical subjects to prohibit, incarcerate or condemn. Rather, it was a positive power that treated people as "working, trading, living beings" (Foucault, 1988, p.156), acting upon them to maximise their potential and productivity. It achieved this through numerous techniques aimed at making individuals' characteristics visible and thus amenable to intervention: a "microscope of conduct" (Foucault, 1979, p.173). New scientific knowledge and techniques of management made it possible to act upon "ordinary individuality," so that each individual might be "described, judged, measured, compared with others, [...] trained, corrected, classified, normalized" (Foucault, 1979, p.191). 
This is an electronic version of an article published in Social Science \& Medicine 99: 80-88. The original publication is available at www.sciencedirect.com. (C) 2013. This manuscript version is made available under the CC-BY-NC-ND 4.0 licence creativecommons.org/licenses/by-nc-nd/4.0/

Most famously, Foucault invoked Jeremy Bentham's plans for the 'Panopticon'. This was a versatile disciplinary institution that, through its architectural design, permitted detailed observation of the individuals within, without the observer being seen, so that the observed come to regulate their own behaviour. The ingenuity of the design facilitated the analytical possibilities described above, but moreover resulted in the internalisation of disciplinary power:

"He who is subject to a field of visibility, and who knows it, assumes responsibility for the constraints of power; he makes them play spontaneously upon himself; he inscribes in himself the power relation in which he simultaneously plays both roles. [...] It is a perpetual victory that avoids any physical confrontation and which is always decided in advance." (Foucault, 1979, pp.202-3)

It was not, though, the Panopticon itself so much as the wider rise of 'panopticism' that interested Foucault. He was concerned with how, from the eighteenth century, disciplinary practices spread beyond institutions to give rise to a 'disciplinary society' that constitutes "an indefinitely generalizable mechanism of 'panopticism', [...]making it possible to bring the effects of power to the most minute and distant elements" (Foucault, 1979, p.216). The result is a society composed of "docile bodies" (Foucault, 1979, pp.135-169): subjects who are properly ordered and normalised, such that they themselves reproduce the logic of their discipline.

\section{Governmentality}

In the late 1970s and early 1980s, Foucault began to trace the contours of a regime of power whose roots lay in the nineteenth century. Propounded largely in lectures, Foucault's notion of governmentality was rather less fully specified than his account of disciplinary power. However, it has since been developed by several scholars to provide an analysis of a regime that has, according to some accounts, displaced the 'disciplinary society' in today's advanced-liberal democracies.

Disciplinary power, and in particular the notion of panopticism, begins to hint at the role of the subject of power in its successful operation. The idea of governmentality goes several steps further. It highlights the importance of a wide range of authorities, and of subjects' own agency, in the workings of power. Nineteenth-century liberal critiques highlighted the limitations of state power (Foucault, 1989); the more effective alternative that emerged in its place was governing through the economy and through society (Foucault, 1981, 1991). The art of governmentality, then, is governing through the freedom of subjects. It involves a power that "incites, it induces, it seduces, it makes easier or more difficult," but it relies above all on influencing "acting subjects" who possess volition, desire and agency (Foucault, 1982, p.220). Where disciplinary power achieves control over subjects (through an external gaze or an internalised awareness of that gaze), governmentality acts upon individuals' very subjectivities, so that it is their own will that guides their actions.

Governmentality provides a lens through which to understand power in complex contemporary societies, highlighting in particular how the state can withdraw from hierarchical imposition by imbuing economistic values into all fields of life to make them self-governing (Rose, 1993). On this view, individuals are 'subjectified' into workers, consumers and citizens who can be entrusted to make the 'right' decisions for themselves. Government 'at a distance', it is argued, through "the entrepreneurial and competitive behaviour of economic-rational individuals" (Foucault, quoted in Lemke, 2001, p.200), offers a more efficient and productive power than sovereign control or the exercise of discipline.

Discipline, governmentality and 'mixed-mode' governance

Governmentality has been much used in analyses of contemporary healthcare organisation, especially in understanding how the increasingly 'arm's-length' relationship between neoliberal states and their healthcare systems of recent decades interacts with already-complicated relationships between professionals, managers and policymakers. This gives rise to novel, but 
functional, regimes of governance, in the field of healthcare quality and safety and elsewhere. Flynn (2002, p.163), for example, characterises the rise of clinical governance as acting upon the professional subjectivities of doctors to make them "accept a responsibility to seek ways of transforming their position themselves," exemplifying the operation of governmentality. Waring (2007) and Levay and Waks (2009, p.512) similarly find quality of care to be achieved most effectively through a subjectification process, where "performance is increasingly assured through continuous self-reporting and self-evaluation, [and] actors and organizations participate in their own governance." More broadly, Sheaff et al. (2004), Ferlie et al. (2012) and Martin and Learmonth (2012) see the shift towards 'network governance' of healthcare as requiring increased investment in 'empowering' and 'responsibilising' professionals to govern themselves. In this way, healthcare governance can rely upon "an entrepreneurial, energetic, quality orientated and value led [...] style, distinct from the neutral affect and routine based style typical of Weberian public bureaucrats" (Ferlie et al., 2012, p.346; cf. Evetts, 2009).

Yet while the increasing reliance on tools of governmentality is well documented, more traditional modes of governing are still evident. Indeed, some argue that professionals are subject to increasing disciplinary power (e.g. Evetts, 2009). Authors have highlighted the panoptic potential of new managerial techniques and developments in IT, which portend greater, more thoroughgoing surveillance of professional activity. Timmons (2003) sees in IT-based nursing training and audit systems the prospect of an 'electronic Panopticon'-though finds that in practice these are easily undermined by nurses themselves, so that their managerial potential goes unrealised. Waring (2007) similarly evokes the spectre of the Panopticon in his analysis of patient safety reporting systems, though again finds them substantially weakened by professional non-cooperation, and thus less effective than governmentality in securing change. At a more general level, Boyne (2000) suggests that panopticism remains highly pertinent to an analysis of contemporary society, despite claims of its redundancy in the era of neoliberal governmentality.

Some argue that disciplinary power and governmentality are contradictory concepts. Rose (1993, p.289), for example, regards governmentality as contrary to disciplinary power's "megalomaniacal and obsessive fantasy of a totally administered society." For Ferlie et al. (2012, p.341) governmentality reflects a "later libertarian" Foucault more concerned with the agency of the subject than with "internalised surveillance." Others are more circumspect, seeing ambiguity and overlap in Foucault's use of terminology and characterisation of regimes (e.g. Elden, 2002). Indeed, Foucault (1988, p.102) himself suggested that regimes of power be understood "not in terms of the replacement of a society of sovereignty by a disciplinary society by a society of government; in reality one has a triangle, sovereignty-discipline-government."

These theoretical debates point towards a key unresolved practical question: how discipline and governmentality might or do interact in complex contemporary governance. Is one more prominent than the other in governing healthcare practitioner behaviour, do they contradict one another, or are there interactions of the kind Foucault (1988) intimated but did not detail? To date, the micro-level impact of both governmentality and discipline has received less attention than the 'macro-physics' of power. With some exceptions (e.g. Ferlie et al., 2012; Iedema, Flabouris, Grant, \& Jorm, 2006; Iedema \& Rhodes, 2010; Martin \& Waring, 2013; Waring, 2007), those drawing on governmentality in particular have been more concerned with the (assumed) power of macro-level governmental discourses and programmes than with their impact on microlevel practice and individual subjectivity (see, e.g., seminal papers on governmentality such as Rose, 1993; or, for a more recent healthcare-related example, Martin \& Learmonth, 2012). Yet it is only in their micro-level impact that we can hope to understand the relative importance of disciplinary power and governmentality in influencing professional behaviour. Thus we ask:

- Is the subjectification of governmentality most influential in governing healthcare practitioners' work?

- $\quad$ Or is it less important than the more coercive approach of disciplinary power, especially 
given the development of new panoptic technologies in the decade since Waring's (2007) and Timmons' (2003) studies took place?

- How does one regime interact with the other?

This paper investigates these issues in a field that has witnessed approaches to governance characteristic of both regimes: healthcare quality and patient safety.

\section{Setting and methods}

Quality and patient safety in healthcare have become prominent concerns, following high-profile reports in the US, UK and elsewhere highlighting how sub-optimal clinical practice results in poor outcomes, wasted resources and harm to patients (Wachter, 2010). Though some encouraging evidence of improvement has been reported in the English National Health Service (NHS) (Benning et al., 2011), large and unexplained variations in quality persist (Hogan et al., 2012), along with high-profile scandals involving egregious failings of individual healthcare providers (e.g. Mid Staffordshire Foundation Trust Public Inquiry, 2013). Limited progress in the field has led opinion leaders to comment on the importance of achieving the right balance of multifaceted approaches, combining technical and social interventions to address the complex challenge of improving quality (e.g. Wachter, 2010).

This paper draws on a wider research programme on quality and safety in the NHS (for an overview, see Dixon-Woods et al., 2013). The study received approval from the Leicestershire, Northamptonshire and Rutland Research Ethics Committee. It included case studies of NHS organisations, sampled for their proactive work addressing quality and safety. Here, we focus on a subsample of three of these organisations, all acute hospitals, chosen because of their use of novel approaches to monitoring the behaviour of staff using specific management techniques and IT-based systems of varying kinds:

- 'Chagley', an acute hospital providing a range of secondary-care services. It had recently introduced a ward-level 'dashboard' system providing up-to-date information on a range of quality and safety-related metrics. It also undertook regular executive walk-arounds, spot checks and internal audits to monitor quality and safety of care.

- $\quad$ 'Thrimpton', a large acute hospital providing secondary- and tertiary-care services. It had developed an electronic decision-support system for medication prescription, and used data from this system to monitor, analyse and manage staff behaviour, through individual-level line management and root-cause analysis meetings when serious adverse events occurred.

- 'Cumberwick Green', a small specialist hospital providing tertiary-care services. It had recently introduced an electronic incident-reporting system for staff to report adverse events and 'near misses', which was then used to analyse risks and evaluate care provided by individuals and teams.

In all cases, senior managers combined these novel approaches with wider efforts to address the socio-cultural dimensions of quality and safety (for example, a patient safety campaign in Chagley, and a 'year of patient safety' in Cumberwick Green), but it was the panoptic potential of the IT systems and associated managerial technologies that was of particular interest in our present analysis.

In all three case studies we conducted interviews with clinical and non-clinical managers, and, though we also undertook ethnographic observations in Chagley and Thrimpton, it is these interviews that are the primary source of data in this article. Interviews took place between February and November 2011. We undertook 19 interviews in Chagley, 11 in Thrimpton and 24 in Cumberwick Green. Interviewees included chief executives, board members, clinical directors and general managers, and practising managers and clinicians in wards and other operating units. These are the kinds of actor who turn high-level policy and organisational concern for quality and safety into strategies to change practitioner behaviour, witness the consequences of such strategies, and adjust them accordingly. They thus provide a partial—but critically important- 
This is an electronic version of an article published in Social Science \& Medicine 99: 80-88. The original publication is available at www.sciencedirect.com. (C) 2013. This manuscript version is made available under the CC-BY-NC-ND 4.0 licence creativecommons.org/licenses/by-nc-nd/4.0/

view on the operation of governance for quality and safety. Interviews covered the place of quality and safety in the organisations, strategies used for improvement, challenges and surmounting them, and specific questions relating to the particular technologies used locally.

Interviews lasted 30-60 minutes and were fully transcribed. GPM led the analysis for this paper, drawing on the constant-comparative method (Charmaz, 2006). He read and reread transcripts, then undertook an initial broad-brush coding of all sections of interviews relating to the governance of quality and safety. Further coding and analysis focused on these sections, involving an iterative process of developing and applying more detailed codes, first around the approaches described in the excerpts, then around what these approaches achieved, where they were limited, and how they interacted. A final stage involved a more theoretical coding process in which these categories were related back to themes identified in writings on discipline and governmentality. Following analysis, the themes and insights were organised into the findings below, which were refined as necessary by all co-authors.

\section{Findings}

We present our findings in three sections. First, we describe how the panoptic and disciplinary potential of new technologies was deployed extensively, and with some efficacy, in these hospitals. Second, we highlight limitations of this approach. We identify the broader strategies used to overcome these limitations, strategies that seemed better conceptualised in terms of governmentality. Finally, we attend more closely to how these approaches coalesced and interacted with one another. Our analysis suggests important interdependencies between the logics of discipline and governmentality-but problematises the mechanisms by which both are conventionally understood to act on subjectivities.

\section{The Panopticon sights back}

In all three hospitals, surveillance of individual-level clinical behaviour formed a key element of the approaches taken to addressing problems of quality and safety. Both the fine-grained application of existing monitoring techniques, and the possibilities created by new IT, provided organisations with a window on individual practice that they were very willing to use. In Chagley, for example, observational audits of frontline staff's practice undertaken by senior staff were used to assess quality of care provided by directorates, wards and individual staff members:

[The auditors] look at aspects around [how] staff introduce themselves, privacy and dignity was maintained, notes were available at all time, results were available — so it's all different aspects of the ward round process that we're looking at. [...] They'll observe-it's an observational audit this-so they'll say, "Yes the staff introduced themselves to the patient, yes the notes were available, yes the results were available," and score it. (Quality Manager 1, Chagley)

In Thrimpton in particular, the electronic prescribing system offered opportunities for individuallevel surveillance that allowed meticulous examination of professionals' practice:

Participant (demonstrating IT system): Each spot represents a nurse. So you've got every single nurse in the organisation. And so here we've got a staff nurse. Over the last six months [she] has been due to give 782 antibiotics. And she's missed 114. And what you can do is click on her, and I'm going to show you a trend just for that nurse over a period of time, whether she's been getting better or whether she's been getting worse.

ML: $\quad$ Good Lord, OK.

Participant: So she's pretty much flat-lined. And then you've got another nurse down here, click on this one. So this is a night sister and she's-

ML: $\quad$-responsible for 1,442. 
This is an electronic version of an article published in Social Science \& Medicine 99: 80-88. The original publication is available at www.sciencedirect.com. (C) 2013. This manuscript version is made available under the CC-BY-NC-ND 4.0 licence creativecommons.org/licenses/by-nc-nd/4.0/

Participant: And she's missed seven. [...] So this nurse is clearly very conscientious, is right on the button in terms of drug administration. But yet then you've got some other people... (Executive Director 1, Thrimpton)

The scope for measuring, comparing with others, correcting and normalising, to paraphrase Foucault (1979), was evident in these hospitals' surveillance systems.

Moreover, managers and senior clinicians were clear that they were willing to use these capacities towards disciplinary (in Foucault's sense) ends. This finding contrasts with Timmons' (2003) report from the 1990 s of reluctance or inability to exploit the disciplinary potential of panoptic IT, and with Waring's (2007) suggestion from the early 2000s that panoptic power was readily resisted by clinicians. In all three hospitals in this study, efforts were made to ensure that information elicited through the various forms of surveillance deployed was used to act upon individual-level clinical practice, including that of doctors as well as nurses:

It's a specific individual we track, if Nurse A keeps making the same mistake again and again. So the ward sisters will also have this information, how many times Nurse A reported an incident, how many times Nurse A was involved in an incident, so you have this record. [...] If there's a particular problem with a member of staff or a certain department, we flag it up. (Senior Quality Manager, Cumberwick Green)

We looked at these unplanned re-attenders and I said, "Let's break it down by individuals and by days and times to see whether there's a particular pattern," and $[\ldots]$ we found that one particular doctor, every time they saw somebody with bellyache and sent them home, that bellyache would come back. [...] We realised that that person didn't have as good skills at assessing bellyaches compared to their peers. (Senior Doctor, Thrimpton)

Unless I'm actually monitoring what individuals are doing and as we've been very clear that this is the standard of practice that we expect, that you just won't get sustainable manageable change. It's about using that process to really get sustained change. (Executive Director 1, Chagley)

Active work was needed to turn panoptic potential into disciplinary efficacy in the practice of individual professionals, and this work had tangible impacts. Ethnographic observation in Thrimpton, for example, suggested strong awareness of the surveillance-oriented use of the prescribing system and fear of the consequences of poor practice; in Cumberwick Green, participants cited improvements in compliance with dress and hand-hygiene codes as evidence of the impact of audits.

There were thus signs that consciousness of the reality of individual-level monitoring was permeating individual clinicians' subjectivities, and translating into a panoptic culture of selfdiscipline. While some complained of 'Big Brother', many accepted that surveillance was an intractable reality, and adjusted their behaviour accordingly to ensure compliance with external expectations:

When it first happened it was like, “Oh, we're being watched," but I suppose I have no problem with it, and people do know, and it's just their way of checking that things are being done. I can report that my hand hygiene is $100 \%$, but obviously on a day when I'm not doing the audit, if someone else comes in, they can double-check. (Senior Nurse 1, Cumberwick Green)

Sometimes you do feel like you are being hit with a big stick, but actually it does make you focus on it and it makes you chase up. [...] That is really positive to be able to say we have changed practice, and we now comply and without doubt improve things." (Quality Manager 2, Chagley)

\section{The limits of panopticism and the role of governmentality}

Despite senior-level enthusiasm for the potential of surveillance and the disciplinary use of the individual-level data produced, and despite signs of its impact on professional behaviour, 
participants also acknowledged the limits of such an approach. Technical limitations were, of course, evident. In all sites, it was recognised that not everything important to quality and safety was amenable to measurement, and that what could be measured required careful interpretation. Evident, also, was reliance on professional acquiescence to the monitoring process: even the granular examination made possible by Thrimpton's high-tech system required that clinicians not share passwords, for example.

Perhaps more importantly, there was recognition (among both the controllers of the systems, and those subjected to them) of the potential unintended consequences of approaches like these. A commonly raised concern was that over-reliance on monitoring systems might undermine professional judgement, diverting attention from less auditable aspects of quality and safety towards those that were measured - the phenomenon known as 'effort redirection' (selfcitation). To this extent, internalisation of the panoptic gaze was potentially problematic. It could constitute the replacement of a self-critical, professional subjectivity with that of an unreflexive rule-follower:

It is making sure they do still know they are responsible... I saw that at a meeting, and the junior doctor obviously had not realised he had done something wrong in [prescribing system], maybe unintentionally, but he had not realised that actually that means you have done a wrong prescription and they could have had a poor outcome because of it. And he said, "Oh well the system allowed me to do that." So it is like over-excusing that it is the system's fault-but no, because you are the doctor and you are the one paid to make the decision. (Senior Quality Manager, Thrimpton)

We've taken away the ability of people to think for themselves and problem-solve, and that really worries me actually. I'm not saying you need to expose people to situations where they feel totally uncomfortable and out of their depth, but sometimes that does people good, in that they have to think in a creative way, they have to problem-solve to get to the solution. (Middle Manager 1, Chagley)

Given these limitations, participants highlighted how for the system of surveillance to work properly, it needed to be accompanied by work on staffs professionalism, in part to encourage them to allow themselves to be subjected to surveillance-but also, perhaps more profoundly, to mediate the influence of surveillance so that its 'proper', intended effects impacted on staff's behaviour while its 'adverse' side-effects did not. Without this, the risk was that the systems would be used to ill-effect, or would only influence those who willingly acceded to them, missing clinicians whose practice was most in need of improvement:

Participant: The anaesthetists didn't like [reporting system], so they put in incident forms which actually I can't do anything about. [...] So they are quite big resisters.

JW: $\quad$ So why do you think they are resisting?

Participant: Because I think they don't have the training, they haven't attended the training, they don't want to attend the training. They are not engaged. [...] It's not an uncommon thing for that particular group of doctors to be resisters: I also work as an infection control nurse, and they were resisters of changes that were brought in, even though there were benefits. (Quality Manager 1, Cumberwick Green)

Securing professional engagement, fundamental to disciplinary power's efficacy, had to be achieved by other means. In each site, therefore, disciplinary work was accompanied by a raft of complementary efforts to create the kind of professional subjects who would 'benefit' optimally from disciplinary use of individual-level knowledge. This was a very different form of influence, underpinned by a very different logic of action upon subjectivities. It was premised not on making individuals' characteristics, deficiencies and potentials visible and amenable to change, but on appealing to professional values, norms and rationalities. In short, it resembled the logic 
of governmentality, treating individuals' freedom to choose as a critical tool for the realisation of objectives, and acting on this freedom by equipping subjects with the right ideals and ambitions to choose wisely.

Three strategies in particular for achieving this subjectifying end were evident in our fieldwork. Professional leadership was one crucial component, with "champions, and juniors learning from seniors" (Senior Doctor 1, Chagley) crucial in establishing quality and safety as a legitimate priority, and establishing the use of individual-level data as a legitimate approach to addressing it:

The chief executive is a healthcare professional, the operations director is a nurse, the medical director is obviously a doctor. So I think they manage to communicate the fact that they have high standards. I think also the positive as well as negative reinforcement is important. So the 'celebrating success' events demonstrate that high-quality care is valued, and giving a good role model as well as making it clear that poor quality care is not tolerated. (Non-executive Director 1, Cumberwick Green)

Second, stories of cases where suboptimal practice had led to harm were an important strategy for securing professional acquiescence to monitoring processes. They helped to make clear that surveillance was not (solely) about accountability and performance management, but also had a direct bearing on patient care:

It's reminding people of the basics, so that's good in itself, it's flagging up that actually we don't get these things right at all and the consequences of that have been quite severe for some patients. So it's making that more real for people: basic observations not done and a patient dies, and the starkness of [how] that can happen in this hospital. (Middle Manager 2, Chagley)

We took our worst-case scenarios and patient stories and we had a patient-well a relative actually — who came in, spoke to the board and then other people, gave this awful story about how it felt as a family member when [her] mother had died in our hospital. It was one of those things where there wasn't absolute error, it wasn't a complete cock-up, but there were a whole load of things which did not make that a good experience. [...] It raised everybody's consciousness that safety applied to everybody. (Senior Doctor 1, Cumberwick Green)

Third, in each hospital, forums existed that brought together professionals to discuss quality and safety and raise their profile. Such forums attempted to act on professional subjectivities, rendering quality and safety a central part of contemporary professionalism, and 'responsibilising' clinicians: "we didn't have any resistance about that—nobody said we didn't need to change" (Middle Manager 3, Chagley). They also worked to generate professional subjectivities that were more amenable to a disciplinary regime of monitoring, analysis and performance management. Thrimpton's root-cause analysis meetings, for example, were ostensibly about identifying underlying issues that had precipitated serious adverse events. A key latent function of the meetings, however, was offering semi-public forums for displaying the consequences of poor-quality care, influencing clinicians in a way that a panoptic regime alone never could:

We had a consultant at the RCA, and she said "I just got that wrong; I just got that completely wrong. I was already prepared to come into this meeting and defend my position but actually now we've gone through all that happened, I've just got it wrong. And I'll make sure that doesn't happen again.” [...] You can guarantee that clinician will not be in front of us for that mistake again. And we are beginning to see that change. If you look at [the intensive care unit] now, every drug chart comes up, first thing that comes up, every patient, the clinicians say to the juniors, "[...] we're going to do everything we're supposed to be doing, 'cause if we do everything we're 
This is an electronic version of an article published in Social Science \& Medicine 99: 80-88. The original publication is available at www.sciencedirect.com. (C) 2013. This manuscript version is made available under the CC-BY-NC-ND 4.0 licence creativecommons.org/licenses/by-nc-nd/4.0/

supposed to be doing, that patient is far more likely to get better, so let's do it." And if we can get that kind of approach, everybody benefits. (Executive Director 2, Thrimpton)

The "choreography" of these forums (Moreira, 2012) was clearly intended to achieve a specific governmental end. Nevertheless, they relied for their efficacy on the way they spoke to professional subjectivities, rather than on providing a window for external surveillance or impelling a docile self-discipline.

Common to all these approaches, then, was that they did not achieve their influence by fiat or managerial diktat. Rather, they found their power in their appeal to professional values and rationality. And in doing so, they created an organisational environment that was more receptive to disciplinary power, and which helped to ensure that its potential adverse consequences were mitigated.

\section{Beyond discipline and subjectification: the depth of the social}

The synergy between disciplinary power and governmentality in these hospitals highlighted their interdependence, and belied any attempt to characterise them simply as antagonistic logics of control. Acting on professional subjectivities through more subtle, governmental modes of influence was crucial if external and internalised disciplinary power was to achieve its positive potential.

Yet on its own, this is to oversimplify the complex relationship between surveillance and subjectivity we found in our data. The panoptic knowledge provided by the monitoring regimes in the three hospitals did not function simply as a means by which individual professionals, rendered more open to the importance of this knowledge by the subjectifying work of governmentality, could be disciplined and discipline themselves to improve their performance. Crucially, it could also serve a more collective-level function.

The disciplining of individuals was only one of the possibilities created by the panoptic regimes of the three hospitals. In making use of the data produced by their various surveillance systems, it became apparent that the level of the individual was not always the most appropriate one for intervention. As Thrimpton's Executive Director 3 noted:

When we fed back the bacteraemia information [...], it didn't change [behaviour]. I think it was because [it] crossed professional groupings and [was] too complex. So the point then was: how do we get people-so for example if there's 20 people involved in patient care, one of them doesn't wash their hands, or if two of them don't wash their hands, do they both cause the bacteraemia? It was too complex, so in the end we had to hold the whole team to account.

In a complex organisational and professional environment, seeking to act solely on individual subjectivity had limited efficacy. Alongside using monitoring data to discipline individuals by holding them to account and providing feedback on their performance, therefore, each hospital also used them in aggregate, at the level of the team, department or ward-with intriguing effects:

[When] the auditors went round, and the results came out, there were a couple of things that weren't right; the results were just abysmally low. People were really, really upset by that, that it was a really bad reflection on their areas, because the other thing is, there is a dashboard, everybody can see everybody's results. We have an infectioncontrol steering group meeting, we all have to go and report on a three-monthly basis about our own individual areas, and everybody [discusses] what is the good practice, what's not been so good, what have our results been like, what are we doing about them, so it's a very transparent thing. The dashboard is there for everybody's results to be seen. (Middle Manager 1, Chagley)

This, then, was not a simple matter of power acting on the individual subject. Rather, it was about the creation of spaces in which clinicians could interact and express their professionalism, 
and thereby envisage and enact new roles and relationships. Sometimes, as in the above quotation, it involved fostering a competitiveness amongst teams that harnessed the professional desire of each to perform as well as possible. At other times, it meant transforming entrenched relationships between professional groups to create a safer culture of critical challenge among professionals who now explicitly shared a stake in each other's performance:

How do we get more collective responsibility for what's going on, whilst maintaining that individual responsibility? I'm as critical of the junior that prescribed badly as I am of the ward tech, pharmacy technician that didn't bother to tell anybody. And you sit there with a drug chart and the ward pharmacy technician is there and you say, "When you looked at this were you happy with that? Were you happy with that drug chart? 'Cause I look at it and I feel ashamed that we've provided that level of care. Now what was your thinking at that point? Didn't you think you should take the doctor to one side? Didn't you think?" We will support constructive challenge in this [hospital]. (Executive Director 2, Thrimpton)

We started just putting up the [hand-hygiene] audit data. Then we split the data out by doctors versus nurses versus other people. And then you could go to the medical staff and say, "How come everybody else can do it and the doctors can't?" Then we start splitting it out by wards. Some of the ward staff were really upset because they were always being pulled down by individuals, often peripatetic individuals like doctors coming and being audited on their ward and messing it up. So we said, "Well, you've got to do something to challenge them then, haven't you?" (Senior Doctor 2, Cumberwick Green)

They didn't always question if it was some other discipline came in. So if a medic came in and didn't do it or a physio, OT, they found it hard to question them. And so that took quite a while to happen properly, [but] it got to the stage where the sisters were all quite happy to say to a consultant, "You haven't rolled your sleeves up. You haven't washed your hands.” (Senior Nurse 2, Cumberwick Green)

What was clear from these accounts was that the panoptic possibilities created by the three hospitals' monitoring systems were not limited to the disciplining of the individual professional subject. Rather, they constituted a resource whose richest potential was in the way it opened new possibilities for collective professional action-for example, inter-professional communication and challenge that is often stymied by hierarchy and professional silo. This was a deeply social use of surveillance data, which accounted for and enhanced professionals' ability to enact their professionalism for the benefit of quality and safety. Its principal mechanism of operation was neither the disciplining of docile subjects through surveillance, nor the responsibilisation of individual subjectivities through the propagation of governmental discourses. On the contrary, it was a process in which the agency of professional subjects was activated, enhanced and inspired by the artful deployment of new knowledge:

I can show you the dashboard. The concept is not new to the organisation, as I've explained. What's new is actually the areas submitting the results [themselves], and that's been a bit challenging but its benefit is that they do take ownership of that, they do realise. I get e-mails now saying, "What audits are due? I know we're supposed to be doing this, when do I need to have it submitted by? It's not on the dashboard, why isn't it on the dashboard, can I see it, where is it?" So it's definitely demonstrating that areas are using it, that wards like it and it's simple as well. We've honed it to a simple tool that areas can use, and they like it. (Quality Manager 1, Chagley)

\section{Discussion: governing the whole?}

Our findings suggest that, in contrast to studies suggesting that 'panoptic' regimes of surveillance 
are readily resisted or subverted by healthcare professionals, at least some hospitals are now deploying managerial techniques and technology to discipline professional behaviour with more impact. We also highlight, though, how this potential rests upon a set of more subtle modes of influence that follow the subjectifying logic of Foucault's notion of governmentality. Despite the evermore penetrating gaze made possible by IT and other managerial techniques, professionals remain able to resist or subvert them. Efforts to make professional subjectivities more receptive to the challenges of quality and safety in general, and to the merit of monitoring professional behaviour in particular, therefore seem critical to the success of such endeavours. It is clear that to avoid the 'failed Panopticons' (Timmons, 2003) of the recent past, such approaches must be accompanied by and founded upon regimes of governmentality. This finding highlights interdependencies between regimes of discipline and governmentality which are sometimes seen as opposed (e.g. Ferlie et al., 2012; Rose, 1993), providing empirical substance to an interdependency intimated by Foucault (1988) himself, and filling out connections that others have suggested (e.g. Waring, 2007). It should also be noted, though, that this realisation is perhaps more co-ordinated and purposive than many readings of Foucault would suggest: there remains some sovereign control and deliberate exploitation of both disciplinary and governmental power by sovereign authority. As Ferlie and McGivern (2013) have argued in their articulation of 'Anglo' governmentality, therefore, sovereign rulers (the state, chief executives) do not relinquish power altogether, but rather "[enrol] a broader range of actors, [use] indirect technologies, and [devolve] operational control but still seeks to retain strategic control" (pp.201). In this light, our findings perhaps illustrate a key means by which managerialism's methods come to be accepted and embraced by professionals, rather than resisted (Numerato et al., 2012).

However, while highlighting the renewed potential of increasingly penetrating panoptic technologies, and governmentality's role in making them efficacious, we also suggest that they do not necessarily operate in the oppressive manner often imagined. Disciplinary power in our study emphatically did not involve the creation of 'docile bodies'; likewise the mechanism by which governmental discourses raised the importance of quality and safety differed from conventional accounts. It did not involve subtle, subliminal work on individuals' subjectivities, as sometimes claimed or implied by studies of those discourses which suggest they have substantial - even constitutive, determinative-power over subjects (Rose, 1993). Rather, the product of the interaction of panopticism and governmentality was the creation of spaces where behaviour change was achieved through novel forms of intra- and inter-professional interaction. This was a thoroughly social form of influence. It operated by prompting new inter-subjective relationships, not by acting on the subject qua rational individual. As such it followed a radically different subjectifying logic to that of disciplinary power and even governmentality, as conventionally understood. The level at which it intervened was not the individual subject, but the collective professional group. It did not represent "a perpetual victory [...] always decided in advance" (Foucault, 1979, p.203), but a starting point from which professionals could explore and negotiate new, undecided opportunities.

There are hints of this logic in some of Foucault's later work. Foucault's (1988) notion of 'technologies of the self highlights the agency involved in fashioning and refashioning subjectivity, an aspect which is sometimes underplayed in contemporary accounts of regimes of governmentality. In such a light, subjectification might be seen less as an involuntary 'imprinting' of values onto subjectivities, and more as a reflexive process involving active consideration of who one wishes to become. Emerging from our findings, though, and from a few other recent studies, are some micro-social processes through which this takes place. The opportunities that arise from discipline and governmentality for individualised resistance or submission are also opportunities for collective creativity and transformation. Iedema and Rhodes (2010, p.212) indicate how even apparently coercive regimes of surveillance beget 'undecided spaces', "where subjects become attentive to their practice and subjectivity and from there generate emergent 
relations with self and other-relations not fully circumscribable or pre-empted by disciplinary power, and not reducible to obedience and resistance." Levay and Waks (2009) and Bejerot and Hasselbladh (2011) similarly intimate that governmentality in professionalised systems is effected not through passive compliance, but through the active, translational work of professionals. This is perhaps one way in which a rising 'organisational professionalism', premised on increasing transparency, accountability and managerial oversight, can interact with more traditional, 'occupational' notions of professionalism (Evetts, 2009). Our findings here cannot be understood simply as a case where "managerial demands for quality control and audit, for target setting and performance review become reinterpreted as promoting professionalism itself" (Evetts, 2009, p.254-5). But they certainly do constitute a rather different notion of professionalism from traditional medical professionalism. Our study illustrates how at the micro level, a complex of governmental and disciplinary power, constitutes a starting point for professional transformation-not a determinate process that either achieves the predefined ends of external authority or is foiled by individual resistance.

Our findings also highlight the need to understand Foucault's regimes of power in the historically informed manner in which he constructed them, especially in contextually idiosyncratic settings such as healthcare. Notions of governmentality and panopticism can be applied glibly and incautiously in understanding the dynamics of contemporary governance, resulting in rather shallow analyses that fail to account for the particularities of historically produced social fields. In healthcare, the distinctive history of professionalism-as a model for autonomous practice, and a mode of social control (Evetts, 2009; self-citation) — would appear to be critical. In this context, the individualising tenets of discipline and governmentality have limited efficacy. A more effective mode of intervention, our findings suggest, is at the level of the social, through appeals to professional values, calls for new expressions of professionalism, and work to reframe intra- and inter-professional relationships. Deploying Foucault's analytical frameworks in ways that do justice to this requires acknowledgement of the historicism of his method, not just application of the concepts that this method produced.

\section{Conclusion}

Our findings lead us to suggest that debates around the relative importance of disciplinary and governmental regimes of power in contemporary governance disregard the mutual dependency of the two forms. We find synergy, not antagonism, between the two, and further suggest that conventional understandings of their mechanisms for effecting behavioural change are incomplete. We highlight instead how, at least in professionalised organisational fields like healthcare, both must engage with existing social forms if they are to achieve their intended objectives. Here lie the practical implications of our study. While prescriptions around quality and safety improvement in healthcare are often divided between those who advocate managerial diktat and those suggesting the need for bottom-up, professionally led solutions (self-citation), crucial in practice is their interdependence and interaction. This is especially notable in relation to managerially mandated expectations about socially mediated behaviours, such as 'speaking up' to challenge colleagues. Future work might further investigate the dynamics of power, resistance and translation in professional contexts, and particularly how these change longitudinally, as disciplinary and governmental regimes continue to develop and become normalised.

\section{Acknowledgements}

We are grateful to the study participants for their candour, to our colleagues on the wider Quality and Safety in the NHS study, and to several colleagues and four anonymous reviewers who provided helpful feedback on earlier drafts of this paper. This project was funded by the Department of Health Policy Research Programme. The views and opinions expressed herein are 
This is an electronic version of an article published in Social Science \& Medicine 99: 80-88. The original publication is available at www.sciencedirect.com. (C) 2013. This manuscript version is made available under the CC-BY-NC-ND 4.0 licence creativecommons.org/licenses/by-nc-nd/4.0/

those of the authors and do not necessarily reflect those of the Policy Research Programme or the Department of Health.

\section{References}

Bejerot,E., \& Hasselbladh,H.(2011).Professional autonomy and pastoral power: the transformation of quality registers in Swedish health care. Public Administration,89(4),16041621.

Benning,A., Dixon-Woods,M., Nwulu,U, Ghaleb,M., Dawson,J., Barber,N. et al. (2011). Multiple component patient safety intervention in English hospitals: controlled evaluation of second phase. BMJ,342,d199.

Boyne,R.(2000).Post-panopticism. Economy and Society,29(2),285-307.

Charmaz,K.(2006).Constructing grounded theory. London: Sage.

Dixon-Woods,M., Baker,R., Charles,K., Dawson,J., Jerzembek,G., Martin,G. et al. (2013). Culture and behaviour in the English National Health Service: overview of lessons from a large multimethod study. BMJ Quality \& Safety published online ahead of print, http://dx.doi.org/10.1136/bmjqs-2013-001947.

Elden,S.(2002).Plague, Panopticon, police. Surveillance \& Society,1(3),240-253.

Ferlie,E., McGivern,G., \& Fitzgerald,L.(2012).A new mode of organizing in health care? Governmentality and managed networks in cancer services in England. Social Science \& Medicine,74(3),340-347.

Flynn,R.(2002).Clinical governance and governmentality. Health, Risk \& Society,4(2),155-173.

Foucault,M.(1979).Discipline and punish. Harmondsworth: Penguin.

Foucault,M.(1981).The history of sexuality (vol.1). Harmondsworth: Penguin.

Foucault,M.(1982).The subject and power. In H.L.Dreyfus \& P.Rabinow (Eds.), Michel Foucault (pp.208-226). Hemel Hempstead: Harvester Wheatsheaf.

Foucault,M.(1988).The political technology of individuals. In L.H.Martin, H.Gutman, \& P.H.Hutton (Eds.), Technologies of the self (pp.145-162). London: Tavistock.

Foucault,M.(1989).An ethics of pleasure. In Foucault live (pp.257-276). New York: Semiotext(e).

Foucault,M.(1991).Governmentality. In G.Burchell, C.Gordon, \& P.Miller (Eds.), The Foucault effect (pp.87-104). Hemel Hempstead: Harvester Wheatsheaf.

Hogan,H., Healey,F., Neale,G., Thomson,R., Vincent,C., \& Black,N.(2012).Preventable deaths due to problems in care in English acute hospitals: a retrospective case record review study. BMJ Quality \& Safety,21,737-745.

Iedema,R., Flabouris,A., Grant,S., \& Jorm,C.(2006).Narrativizing errors of care: critical incident reporting in clinical practice. Social Science \& Medicine,62(1),134-144.

Iedema,R., \& Rhodes,C.(2010).The undecided space of ethics in organizational surveillance. Organization Studies,31(2),199-217.

Lemke,T.(2001).“The birth of bio-politics”: Michel Foucault's lecture at the Collège de France on neo-liberal governmentality. Economy and Society,30(2),190-207.

Levay,C., \& Waks,C.(2009).Professions and the pursuit of transparency in healthcare: two cases of soft autonomy. Organization Studies,30(5),509-527.

Martin,G.P., Armstrong,N., Aveling,E.-L., Herbert,G., \& Dixon-Woods,M. (2012). Professionalism reinvigorated or redundant? Harnessing clinical communities to improve healthcare quality. British Sociological Association Medical Sociology Association $44^{\text {th }}$ Annual conference, Leicester, United Kingdom.

Martin,G.P., \& Learmonth,M. (2012). A critical account of the rise and spread of 'leadership': the case of UK healthcare. Social Science \& Medicine,74(3),281-288.

Martin,G.P., \& Waring,J. (2013). Leading from the middle: constrained realities of clinical leadership in healthcare organisations. Health,17(4),358-374.

Mid Staffordshire Foundation Trust Public Inquiry (2013).Report of the Mid Staffordshire Foundation 
This is an electronic version of an article published in Social Science \& Medicine 99: 80-88. The original publication is available at www.sciencedirect.com. (C) 2013. This manuscript version is made available under the CC-BY-NC-ND 4.0 licence creativecommons.org/licenses/by-nc-nd/4.0/

Trust Public Inquiry. www.midstaffspublicinquiry.com/report.

Moreira,T.(2012). The transformation of contemporary health care. London: Routledge.

Numerato,D., Salvatore,D., \& Fattore,G.(2012).The impact of management on medical professionalism: a review. Sociology of Health \& Illness, 34(4),626-644.

Rhodes,R.A.W.(2007).Understanding governance: ten years on. Organization Studies,28(8),12431264

Rose,N.(1993).Government, authority and expertise in advanced liberalism. Economy and Society,22(3),283-299.

Sheaff,R., et al.(2004).Governmentality by network in English primary healthcare. Social Policy \& Administration,38(1),89-103.

Timmons,S.(2003).A failed panopticon: surveillance of nursing practice via new technology. New Technology, Work and Employment,18(2),143-153.

Wachter,R.M.(2010).Patient safety at ten: unmistakable progress, troubling gaps. Health Affairs,29(1),165-173.

Waring,J.(2007).Adaptive regulation or governmentality: patient safety and the changing regulation of medicine. Sociology of Health \& Illness,29(2),163-179. 